\title{
Assessment of the Efficacy of Various Subgingival Irrigating Solutions in Chronic Periodontitis: A Comparative Study
}

\author{
Mohammad Jalaluddin ${ }^{1}$, Shilpa Mailankote ${ }^{2}$, George Sam $^{3}$, Narendra V Penumatsa ${ }^{4}$, Abdulfatah Alazmah $^{5}$, Prashant Punde $^{6}$
}

\begin{abstract}
Aim: The present study aimed to evaluate the efficacy of different subgingival irrigating solutions, which can be used in the treatment of chronic periodontitis.

Materials and methods: Altogether 60 patients (both females and males) aged between 20 years and 60 years who were diagnosed with mild to moderate chronic periodontitis were chosen for this study. After providing oral hygiene maintenance instructions, the patients were grouped randomly divided into three divisions. Group I: subgingival irrigation with tetracycline $\mathrm{HCl} 10 \mathrm{mg} / \mathrm{mL}$, group II: subgingival irrigation with $0.2 \%$ chlorhexidine, group III: Subgingival irrigation with $10 \%$ povidone-iodine. The subgingival plaque samples were collected and clinical parameters such as probing pocket depth, gingival index, plaque index, and clinical attachment level were recorded at the selected sites at baseline, after 15 days and at the end of 1 month.

Results: The mean gingival index score decreased from $1.76 \pm 0.10$ at baseline to $1.07 \pm 0.01$ after 1 month of irrigation, and the mean plaque index score decreased from $1.52 \pm 0.20$ at baseline to $1.10 \pm 0.71$ after 1 month of irrigation in group I. A statistically significant difference was found on intergroup comparisons of clinical attachment level, pocket probing depth, and colony-forming units.

Conclusion: In conclusion, a significant improvement in the clinical parameters was seen in all the three investigational groups in our study. Nevertheless, slightly better improvement in clinical parameters was demonstrated by the tetracycline $\mathrm{HCl}$ irrigation group when compared to povidone-iodine and chlorhexidine irrigating solutions.

Clinical significance: The use of subgingival irrigating solutions interferes with the development of the intricate ecosystem, which is required for the commencement and sustained destruction of the periodontium in any vulnerable host. The administration of antimicrobial agents locally offers a "site-precise" method of periodontal treatment and it has numerous advantages.

Keywords: Chlorhexidine, Chronic periodontitis, Povidone-iodine, Subgingival irrigation, Tetracycline $\mathrm{HCl}$.

World Journal of Dentistry (2020): 10.5005/jp-journals-10015-1735
\end{abstract}

\section{INTRODUCTION}

A chronic inflammatory condition of structures that support teeth is known as periodontitis, which gradually progresses to tooth loss. The chief etiological factor for periodontitis is dental plaque. Most of the periodontal investigations are focused around finding more efficient and cost-effective ways of managing dental plaque and consequent disease progression. ${ }^{1}$

The procedure of scaling and root planing (SRP) is the historical treatment method, which is considered as the "gold standard" among the nonsurgical methods of periodontal disease management. Nevertheless, SRP has some demerits such as failure of instrument to reach the bifurcations and deeper periodontal pockets, and it could not eliminate the microorganisms along the periodontal pocket lining. Thus, antimicrobial agents target the vulnerable microorganisms present in different oral cavity habitats. ${ }^{2}$

The success of local antimicrobial agents used in periodontal management rests on delivery of the agent subgingivally, maintenance of active concentrations of the antimicrobial agent, and achieving enough contact time between target microorganisms and the antimicrobial agent. Inability to achieve at least one of these parameters could be the cause for the comparative ineffectiveness of several antimicrobial treatments applied locally in periodontics. ${ }^{3}$

Lack of acceptable levels of subgingival delivery of antimicrobial agents by different local drug delivery systems is a challenging part of periodontal treatment. The antiseptics present in mouth rinse have no direct effects on the microorganisms present subgingivally
${ }^{1}$ Department of Periodontics and Oral Implantology, Kalinga Institute of Dental Sciences, KIIT University, Bhubaneswar, Odisha, India

${ }^{2}$ Department of Public Health Dentistry, AB Shetty Memorial Institute of Dental Sciences, NITTE (Deemed to be University), Deralakatte, Mangaluru, Karnataka, India

${ }^{3-5}$ Department of Preventive Dental Sciences, College of Dentistry, Prince Sattam Bin Abdul Aziz University, Al-Kharj, Kingdom of Saudi Arabia

${ }^{6}$ Department of Oral and Maxillofacial Surgery, School of Dental Sciences, Krishna Institute of Medical Sciences (Deemed to be University), Karad, Maharashtra, India

Corresponding Author: Mohammad Jalaluddin, Department of Periodontics and Oral Implantology, Kalinga Institute of Dental Sciences, KIIT University, Bhubaneswar, Odisha, India, Phone: +91 9338131843, e-mail: drjalal1979@gmail.com

How to cite this article: Jalaluddin M, Mailankote S, Sam G, et al. Assessment of the Efficacy of Various Subgingival Irrigating Solutions in Chronic Periodontitis: A Comparative Study. World J Dent 2020;11(3):221-225.

Source of support: Nil

Conflict of interest: None

because of the almost complete absence of diffusion of mouth rinse beyond the gingival margin (mean $0.2 \mathrm{~mm}$ ). Likewise, toothbrushes do not reach substantial areas of the subgingival region (mean $0.9 \mathrm{~mm}$ penetration). ${ }^{4}$

() The Author(s). 2020 Open Access This article is distributed under the terms of the Creative Commons Attribution 4.0 International License (https://creativecommons. org/licenses/by-nc/4.0/), which permits unrestricted use, distribution, and non-commercial reproduction in any medium, provided you give appropriate credit to the original author(s) and the source, provide a link to the Creative Commons license, and indicate if changes were made. The Creative Commons Public Domain Dedication waiver (http://creativecommons.org/publicdomain/zero/1.0/) applies to the data made available in this article, unless otherwise stated. 
Over the past 40 years, subgingival irrigation has been an effective adjunct in periodontal treatment. The success of both in office and at home periodontal treatment relies on delivery of antimicrobial agents subgingivally. In the recent past, several antibacterial agents, for example, chlorhexidine $(\mathrm{CHX})$, tetracyclines, metronidazole, and povidone-iodine and other herbal products such as dentol and propolis ${ }^{5}$ have been investigated and have been demonstrated to be effective subgingival irrigants in the management of periodontal pockets that are not deep. Nevertheless, there is ongoing research to explore other drugs that are more effective than those currently used. Thus, this present study was conducted with an objective to evaluate the efficacy of various subgingival irrigating solutions in chronic periodontitis.

\section{Materials and Methods}

The present study was conducted in the Department of Periodontics, Kalinga Institute of Dental Sciences, Bhubaneswar, Odisha, India. Altogether 60 patients (both females and males) aged between 20 years and 60 years diagnosed with mild to moderate type of chronic periodontitis were chosen for the study and a written informed consent was obtained from each patient.

\section{Patient Selection Criteria}

Patients having at least $5 \mathrm{~mm}$ periodontal probing depth, bleeding on probing (BOP), and at least one periodontal pocket in two or more different quadrants were included. Patients had to be nonsmokers with $\geq 20$ natural teeth and had not received any periodontal treatment in the previous 6 months. Patients who had received any antibiotics or any form of periodontal treatment in the last 6 months, teeth with overhanging restorations, past systemic disease that could impact the periodontal disease course or would require prophylactic antibiotics before dental treatment, pregnant, and allergic to the drugs being used were excluded from the study.

\section{Study Design}

The structure and design of the study was briefed to all the patients in their local language and a written informed consent was taken from each patient. An instruction to maintain oral hygiene and control supragingival plaque was given to all patients. Each participant was guided to brush two times a day with a soft-bristled toothbrush and toothpaste based on the Bass method. Later, the patients were randomly grouped into three divisions.

\section{Group I: Subgingival Irrigation with Tetracycline $\mathrm{HCl} 10 \mathrm{mg} / \mathrm{mL}$}

Three tetracycline $\mathrm{HCl}$ capsules of $500 \mathrm{mg}$ (Tetraclin $500 \mathrm{mg}$ ) strength each were dissolved in $150 \mathrm{~mL}$ double-distilled sterile water to obtain tetracycline solutions. Subgingival tips of a mechanical irrigator were used to generate an exciting jet of the irrigating solution and deposit agent subgingivally. The surplus irrigating solution was aspirated continuously. The irrigation procedure was performed for about 5 minutes along each chosen site.

\section{Group II: Subgingival Irrigation with $0.2 \%$ Chlorhexidine}

A Waterpik irrigator device with $0.2 \%$ chlorhexidine gluconate (Clohex Mouth Wash) placed in its reservoir was used. The Waterpik irrigator was established and irrigation was performed for about 5 minutes along each chosen site.

\section{Group III: Subgingival Irrigation with 10\% Povidone-iodine}

A double side-vented endodontic syringe was used to irrigate $1 \mathrm{~mL}$ of $10 \%$ povidone-iodine solution (Wokadine $10 \%$ Solution). The syringe was mildly introduced into the periodontal pockets to its complete depth to ensure delivery of irrigating solution. Reirrigation confirmed that irrigating solution fills up periodontal pockets for about 5 minutes and instructions to maintain oral hygiene were reiterated.

\section{Clinical Examination}

Instructions to maintain oral hygiene and SRP procedures were performed 1 week before initiation of the trial. Instrumentation was not done on experimental teeth and teeth that are immediately adjacent to it. The clinical parameters such as probing pocket depth, gingival index, plaque index, and clinical attachment level were noted from the selected sites and samples of the subgingival plaque were collected at baseline, after 15 days and at the end of 1 month.

\section{Plaque Sample Collection for Colony-forming Units Count}

At baseline, after 15 days, and at the end of the 1 month, plaque samples were collected. Cotton rolls were used for isolation of the site and the supragingival plaque was collected with sterile curettes and subgingival plaque samples were collected from the deepest periodontal pockets before the initiation of periodontal treatment. Later, three paper points were consecutively placed into the periodontal pocket depths for 10 seconds each to yield the subgingival plaque. After removal of paper points, the $3 \mathrm{~mm}$ of each point toward the apical end was instantly placed into a centrifuge tube having sterile saline of $2 \mathrm{~mL}$ quantity (transport media) and kept in kanamycin blood agar as the anaerobic selective medium. $P$. gingivalis count was done at baseline, after 15 days, and at the end of the 1 month in all the three groups.

\section{Statistical Analysis}

The mean and standard deviations were calculated using the SPSS software of version 17.0. The comparisons within and between and different irrigating solution groups were assessed using the one-way analysis of variance (ANOVA). A $p$ value of less than 0.05 was considered statistically significant.

\section{Results}

Table 1 shows the comparisons between the gingival index at baseline, 15 days, and 1 month. The mean gingival index score was $1.76 \pm 0.10$ at baseline, $1.10 \pm 0.34$ after 15 days, and $1.07 \pm 0.01$ after 1 month in group I. The mean gingival index score reduced from $1.68 \pm 0.02$ at baseline to $1.18 \pm 0.17$ after 1 month in group II. Similar reductions in the mean gingival index score was seen in group III, reducing to $1.32 \pm 0.11$ after 1 month from $1.64 \pm 0.32$ at baseline. All the three groups demonstrated statistically significant difference at various intervals.

The plaque index at baseline, 15 days, and after 1 month is as displayed by Table 2 . In group I, the mean plaque index score reduced from $1.52 \pm 0.20$ at baseline to $1.10 \pm 0.71$ after 1 month. The mean plaque index score reduced to $1.08 \pm 0.27$ after 1 month from $1.49 \pm 0.43$ at baseline in group II. In group III, the reduction in mean plaque index score was $1.26 \pm 0.05$ at the end of 1 month from $1.56 \pm 0.16$ at baseline. A statistically significant difference was seen at various intervals in all the three groups. 
Table 1: Comparison of gingival index at baseline, 15 days, and after 1 month

\begin{tabular}{|c|c|c|c|c|c|}
\hline & Groups & & Mean $\pm S D$ & Fvalue & $p$ value \\
\hline \multirow[t]{9}{*}{ Gingival index } & Group I & Baseline & $1.76 \pm 0.10$ & 7.160 & 0.001 \\
\hline & & 15 days & $1.10 \pm 0.34$ & & \\
\hline & & 1 month & $1.07 \pm 0.01$ & & \\
\hline & Group II & Baseline & $1.68 \pm 0.02$ & 8.249 & 0.001 \\
\hline & & 15 days & $1.22 \pm 0.14$ & & \\
\hline & & 1 month & $1.18 \pm 0.17$ & & \\
\hline & Group III & Baseline & $1.64 \pm 0.32$ & 9.182 & 0.001 \\
\hline & & 15 days & $1.30 \pm 0.28$ & & \\
\hline & & 1 month & $1.32 \pm 0.11$ & & \\
\hline
\end{tabular}

Table 2: Comparison of plaque index at baseline, 15 days, and after 1 month

\begin{tabular}{|c|c|c|c|c|c|}
\hline & Groups & & Mean $\pm S D$ & Fvalue & $p$ value \\
\hline \multirow[t]{9}{*}{ Plaque index } & Group I & Baseline & $1.52 \pm 0.20$ & 9.167 & 0.001 \\
\hline & & 15 days & $0.98 \pm 0.12$ & & \\
\hline & & 1 month & $1.10 \pm 0.71$ & & \\
\hline & Group II & Baseline & $1.49 \pm 0.43$ & 8.322 & 0.001 \\
\hline & & 15 days & $1.04 \pm 0.08$ & & \\
\hline & & 1 month & $1.08 \pm 0.27$ & & \\
\hline & Group III & Baseline & $1.56 \pm 0.16$ & 8.221 & 0.001 \\
\hline & & 15 days & $1.18 \pm 0.28$ & & \\
\hline & & 1 Month & $1.26 \pm 0.05$ & & \\
\hline
\end{tabular}

Table 3: Comparison of probing pocket depth at baseline, 15 days, and after 1 month

\begin{tabular}{|c|c|c|c|c|c|}
\hline & Groups & & Mean $\pm S D$ & Fvalue & $p$ value \\
\hline \multirow[t]{9}{*}{ Probing pocket depth } & Group I & Baseline & $5.20 \pm 0.13$ & 10.120 & 0.001 \\
\hline & & 15 days & $4.10 \pm 0.18$ & & \\
\hline & & 1 month & $3.60 \pm 0.09$ & & \\
\hline & Group II & Baseline & $5.38 \pm 0.70$ & 12.268 & 0.001 \\
\hline & & 15 days & $4.60 \pm 0.18$ & & \\
\hline & & 1 month & $4.28 \pm 0.02$ & & \\
\hline & Group III & Baseline & $5.64 \pm 0.66$ & 9.814 & 0.001 \\
\hline & & 15 days & $4.86 \pm 0.24$ & & \\
\hline & & 1 month & $3.98 \pm 0.32$ & & \\
\hline
\end{tabular}

In group I, the mean value for probing pocket depth of 5.20 \pm 0.13 at baseline reduced to $3.60 \pm 0.09$ after 1 month. Likewise, reductions in the mean values for pocket depth probing were noted from $5.38 \pm 0.70$ to $4.28 \pm 0.02$ in group II, and from $5.64 \pm 0.66$ to $3.98 \pm 0.32$ in group III. Intergroup comparisons of the probing pocket depth demonstrated statistically significant difference (Table 3).

The mean values for the clinical attachment level are as shown in Table 4. A reduction from $5.10 \pm 0.42$ at baseline in clinical attachment to $3.65 \pm 0.10$ after 1 month was noted for group I. Similar decrease in clinical attachments from $5.08 \pm 0.52$ to 3.89 \pm 0.14 and $5.02 \pm 0.18$ to $4.08 \pm 0.14$ were seen in groups II and III, respectively. A statistically significant intergroup comparison of the clinical attachment level was found.

Figure 1 demonstrates the statistically significant decrease in colony-forming units in all the three groups at the end of 1 month. A reduction from $3.22 \pm 0.12$ at baseline in colony count to $1.45 \pm$ 0.26 after 1 month was noted for group I. Similar decrease in colony count from $3.10 \pm 0.40$ to $1.56 \pm 0.06$ and $3.46 \pm 0.28$ to $1.85 \pm 0.02$ was seen in groups II and III, respectively.

The inference of the present study indicates that the tetracycline $\mathrm{HCl}$ irrigation group is significantly more efficient in improving all the clinical parameters and reduction of colony count compared to povidone-iodine and chlorhexidine irrigating solution groups, respectively.

\section{Discussion}

The chief etiologic causes of periodontal disease are definite bacterial dental plaques and their products, which are active biologically. The important goal of periodontal management is elimination and control of dental plaque and restoration of common bacterial organisms in the sulcus of gingiva. The periodontal treatment usually relies upon maintenance of oral hygiene and debridement of root surfaces to reduce the periodontal flora. ${ }^{6}$

Therefore, to enhance the special effects of SRP, either topical or systemic antibiotics or local irrigating solutions with a range 
Table 4: Comparison of clinical attachment level at baseline, 15 days, and after 1 month

\begin{tabular}{|c|c|c|c|c|c|}
\hline & Groups & & Mean $\pm S D$ & Fvalue & $p$ value \\
\hline \multirow{9}{*}{$\begin{array}{l}\text { Clinical attachment } \\
\text { level }\end{array}$} & \multirow[t]{3}{*}{ Group I } & Baseline & $5.10 \pm 0.42$ & \multirow[t]{3}{*}{10.228} & \multirow[t]{3}{*}{0.001} \\
\hline & & 15 days & $4.12 \pm 0.22$ & & \\
\hline & & 1 month & $3.65 \pm 0.10$ & & \\
\hline & \multirow[t]{3}{*}{ Group II } & Baseline & $5.08 \pm 0.52$ & \multirow[t]{3}{*}{9.619} & \multirow[t]{3}{*}{0.001} \\
\hline & & 15 days & $4.50 \pm 0.12$ & & \\
\hline & & 1 month & $3.89 \pm 0.14$ & & \\
\hline & \multirow[t]{3}{*}{ Group III } & Baseline & $5.02 \pm 0.18$ & \multirow[t]{3}{*}{10.168} & \multirow[t]{3}{*}{0.001} \\
\hline & & 15 days & $4.72 \pm 0.90$ & & \\
\hline & & 1 month & $4.08 \pm 0.14$ & & \\
\hline
\end{tabular}

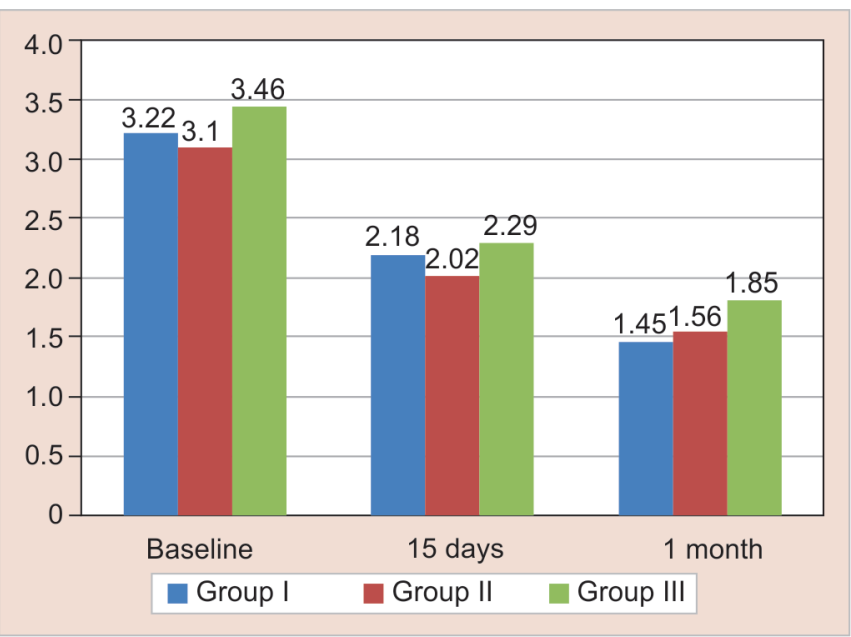

Fig. 1: Comparison of colony count at baseline, 15 days, and after 1 month

of antimicrobial agents have been used. The advancement of chemotherapeutic agents able to hinder the formation of dental plaque has been of important significance to practicing dentists and dental researchers since the last decade. The containment of pathogens from periodontal tissues can be achieved with the application of antimicrobial agents locally, and this improves the microbial and clinical advantage of mechanical debridement, however with its demerits and side effects such as taste alterations, teeth staining, etc. ${ }^{7}$

The clinical parameters like probing pocket depth, gingival index, plaque index, and clinical attachment level significantly improved in the present study after 1 month in group I (tetracycline irrigation), indicating the benefits of adjunctive subgingival tetracycline irrigation. This could be attributed to the antibacterial roles of tetracycline when used for subgingival irrigation. As per Silverstein et al. ${ }^{8}$ when a waterpik is used to deliver tetracycline subgingivally, an increased levels of tetracycline is obtained within gingival crevicular fluid and these levels are more than that achieved with antibiotics administered systemically. Additionally, tetracycline has been shown to be efficacious against the periodontal diseasecausing pathogenic microorganisms, by increasing the attachment of fibroblasts to fibronectin-associated dental structures and by inhibiting the activity of collagenase. Furthermore, the important benefit of delivery of tetracycline- $\mathrm{HCl}$ locally into periodontal pockets when compared to systemic administration is the increased concentration of the drug locally at the areas of increased disease activity with negligible effects on the microorganisms present elsewhere.

This study is in accord with Stabholz et al. ${ }^{9}$ and Krishna et al. ${ }^{10}$ who assessed the use of a single period of tetracycline $(10 \mathrm{mg} /$ $\mathrm{mL}$ ) irrigation with no SRP at the investigational sites. As per their study, the extent of antimicrobial activity achieved is comparative to the tetracycline $\mathrm{HCl}$ concentration used for irrigation. The SRP procedure was performed before the start of their study, without including the study sites. Likewise in the present study, a low dose of tetracycline $(10 \mathrm{mg} / \mathrm{mL})$ was locally delivered at the site that was affected.

In the present study, $10 \%$ povidone-iodine irrigation demonstrated a significant fall in clinical parameters such as PPD, PI, $\mathrm{Gl}$, and CAL. This could be attributed to the use of povidone-iodine irrigating solutions adjunctively to maintain the antimicrobial effect for a longer period of time. The results of our study were similar to those achieved previously by Pandya et al. ${ }^{11}$ and Sindhura et al. ${ }^{12}$ An expected decrease in the inflammation levels and no BOP were also demonstrated. The measured subgingival iodine release stimulates the new influx of T-helper cells and macrophages, which assist in healing of wounds and also reduces the inflammation as shown by Selvaggi et al. ${ }^{13}$

Chlorhexidine has come into view as an adjunct to periodontal treatment and as a chief antibacterial oral agent. It has a universal antiseptic effect with distinct antimicrobial effects on bacteria (both gram-positive and gram-negative), a few viruses, and fungi. The combination use of chlorhexidine and irrigating solutions has emerged to be more efficacious at changing the microflora subgingivally when compared to use of mouth rinse alone. Also, a significant decrease in spirochetes and motile organisms has been noted after every day irrigation with chlorhexidine $0.2 \%$ in comparison with a single-sitting SRP and rendering of oral hygiene instructions. ${ }^{14}$

In this study, PI, GI, PPD, and CAL reduced significantly with the use of $0.2 \% \mathrm{CHX}$ irrigation. This is in agreement with the results obtained by Nezih et al., ${ }^{15}$ Heasman et al., ${ }^{16}$ Mizrak et al. ${ }^{17}$ The capability of treatment regimens to reduce the mean increased depth of pockets to a maintainable healthy pocket depth was noted for sites with a pocket depth of 4-7 $\mathrm{mm}$ at baseline.

The limitation of this study was that the sample size was small and the time period to evaluate the efficiency of the investigational irrigating solution was short. Therefore, longitudinal studies with bigger sample size and extensive follow-up period are recommended in future to assess the long-term benefits of regular use and influence of these irrigating solutions on other periodontal pathogenic organisms in chronic periodontitis. 


\section{Conclusion}

In conclusion, a significant improvement in the clinical parameters was seen in all the three investigational groups in our study. Nevertheless, slightly better improvement in clinical parameters was demonstrated by the tetracycline $\mathrm{HCl}$ irrigation group when compared to povidone-iodine and chlorhexidine irrigating solutions.

\section{References}

1. Singhla R, Tevatia S, Chaudhry S, et al. Comparative evaluation of the efficacy of locally delivered $0.1 \%$ curcumin irrigation, meswak irrigation and $0.2 \% \mathrm{CHX}$ irrigation in management chronic periodontitis patients. J Dent Specialities 2017;5(1):53-57.

2. Cobb CM. Microbes, inflammation, scaling and root planing, and the periodontal condition. J Dent Hyg 2008;82(Suppl 3):4-9.

3. Shewale A, Gattani D, Bhasin MT, et al. Adjunctive role of supra- and subgingival irrigation in periodontal therapy. Int J Pharm Sci Res 2016;7(3):152-159.

4. Wunderlich RC, Singleton M, O'Brien WJ, et al. Subgingival penetration of applied solutions. Int J Periodontics Restorative Dent 1984;6:65-71.

5. Shahab A, Haghighati F, Baeeri M, et al. A clinical, microbiological and immunological comparison between subgingival irrigation with dentol and chlorhexidine in advanced periodontitis. Arch Med Sci 2011;7(1):154-160. DOI: 10.5114/aoms.2011.20622.

6. Abullais SS, Dani N, Hamiduddin, et al. Efficacy of irrigation with different antimicrobial agents on periodontal health in patients treated for chronic periodontitis: a randomized controlled clinical trial. Ayu 2015;36(4):380-386. DOI: 10.4103/0974-8520.190702.

7. Hallmon WW, Tery DR. Local anti-infective therapy: mechanical and physical approaches: a systematic review. Ann Periodontol 2003;8(1):99-114. DOI: 10.1902/annals.2003.8.1.99.

8. Silverstein L, Bissada N, Manouchehr-Pour M, et al. Clinical and microbiologic effects of local tetracycline irrigation on periodontitis.
J Periodontol 1988;59(5):301-305. DOI: 10.1902/jop.1988. 59.5.301.

9. Stabholz A, Nicholas AA, Zimmerman GJ, et al. Clinical and antimicrobial effects of a single episode of subgingival irrigation with tetracycline $\mathrm{HCl}$ or chlorhexidine in deep periodontal pockets. J Clin Periodontol 1998;25(10):794-800. DOI: 10.1111/j.1600-051X.1998. tb02372.x.

10. Krishna MK, Ravindran SK, Vivekanandan G, et al. Effects of a single episode of subgingival irrigation with tetracycline $\mathrm{HCl}$ or chlorhexidine: a clinical and microbiological study. J Indian Soc Periodontol 2011;15(3):245-249. DOI: 10.4103/0972-124X.85668.

11. Pandya D, Manohan B, Darshan V, et al. Povidone iodine - an adjunct to periodontal therapy. NJIRM 2012;3:148-151.

12. Sindhura $H$, Harsha $R H$, Shilpa RH. Efficacy of subgingival irrigation with $10 \%$ povidone-iodine as an adjunct to scaling and root planing: a clinical and microbiological study. Indian J Dent Res 2017;28(5): 514-518. DOI: 10.4103/ijdr.IJDR_497_15.

13. Selvaggi G, Monstrey S, Van Landuyt K, et al. The role of iodine in antisepsis and wound management: a reappraisal. Acta Chir Belg 2003;103(3):241-247. DOI: 10.1080/00015458.2003.11679417.

14. Kshitish D, Laxman VK. The use of ozonated water and $0.2 \%$ chlorhexidine in the treatment of periodontitis patients: a clinical and microbiologic study. Indian J Dent Res 2010;21(3):341-348. DOI: 10.4103/0970-9290.70796.

15. Nezih A, Gul A, Hanne L, et al. The effect of subgingival controlled release delivery of chlorhexidine chip on clinical parameters and matrix metalloproteinases-8 level in gingival crevicular fluid. J Periodontol 2002;73(6):605-615.

16. Heasman PA, Heasman L, Stacey F, et al. Local delivery of chlorhexidine gloconate (periochip) in periodontal maintenance patients. J Clin Periodontol 2001;28(1):90-95. DOI: 10.1034/j.1600051x.2001.280114.x.

17. Mizrak T, Güncü GN, Caglayan F, et al. Effect of a controlled-release chlorhexidine chip on clinical and microbiological parameters and prostaglandin E2 levels in gingival crevicular fluid. J Periodontol 2006;77(3):437-443. DOI: 10.1902/jop.2006.050105. 